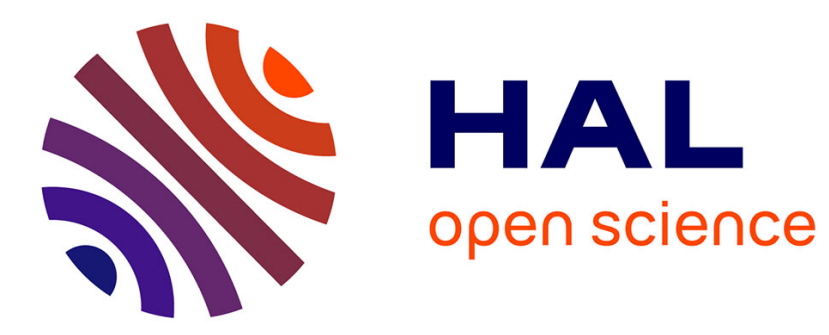

\title{
Multi-phase System Supplied by SVM VSI: A New Fast Algorithm to Compute Duty Cycles
}

\author{
Xavier Kestelyn, Eric Semail, Jean-Paul Hautier
}

\section{To cite this version:}

Xavier Kestelyn, Eric Semail, Jean-Paul Hautier. Multi-phase System Supplied by SVM VSI: A New Fast Algorithm to Compute Duty Cycles. Multi-phase System Supplied by SVM VSI: A New Fast Algorithm to Compute Duty Cycles, 2004, 14 (3), pp.1-11. hal-00820349

\section{HAL Id: hal-00820349 https://hal.science/hal-00820349}

Submitted on 3 May 2013

HAL is a multi-disciplinary open access archive for the deposit and dissemination of scientific research documents, whether they are published or not. The documents may come from teaching and research institutions in France or abroad, or from public or private research centers.
L'archive ouverte pluridisciplinaire HAL, est destinée au dépôt et à la diffusion de documents scientifiques de niveau recherche, publiés ou non, émanant des établissements d'enseignement et de recherche français ou étrangers, des laboratoires publics ou privés. 


\title{
Multi-phase System Supplied by SVM VSI: \\ A New Fast Algorithm to Compute Duty Cycles
}

\author{
Xavier Kestelyn, Eric Semail, J.P Hautier. \\ L2EP, Laboratoire d'Electrotechnique et d' Electronique de Puissance de Lille \\ ENSAM Lille 8 bd Louis XIV F-59046 LILLE, France \\ e-mail: Xavier.Kestelyn@univ-lille1.fr \\ http://www.univ-lille1.fr/12ep/
}

Keywords: Multi-phase, Space Vector Modulation, Fast Algorithm

\section{Summary}

Multi-phase drives are more and more often used in industry, especially for high-power applications [17] [18] [20]. Space Vector Modulation (SVM) is now widely implemented and possesses many advantages over carrier-based pulse width modulation (PWM):

- Natural overmodulation implementation

- Easy solution for saturation treatment

- Fast and convenient to compute

- Easy implementation of switching constraints for example to reduce harmonic currents [19].

Many authors proposed SVM VSI applied to multi-phase drives. For example, [2] and [15] have chosen instantaneous vectors to control dual 3-phase induction machines with low generated harmonic currents, [4] and [6] to control 5-phase machines. The initial space is split onto orthogonal subspaces (d-q and zero-sequence) and the initial reference vector can be expressed at any sampling time in terms of several reference vectors, each one belonging to one subspace (plane and/or line). Each reference vector is located onto a sector, bounded by two active vectors, and decomposed onto these vectors. Once the two vector components are known, duty ratios are determined. Then, zero vectors are chosen and switching sequencing is imposed. Due to the high number of phases, a high number of sectors exist and the location of the different reference vectors leads to a great computational requirement (Fig. 1a).

Using the equivalence between a multi-phase machine and a set of fictitious one-phase or twophase machines which are magnetically independent but mechanically and electrically coupled [1][13], we propose a new fast algorithm to compute the duty cycles of each VSI leg. This algorithm, based on a vectorial approach of inverters developed in [3][7][5], thereby reduces computation time and allows to use low computational requirements. This paper shows that, compared to classical technics [11] [10] [9] [12], it is no more necessary to find the location of the reference vectors to get explicitly the duty cycle of each leg. Figure 1 shows the difference between classical algorithm (Fig. 1a) and proposed one (Fig. 1b).

This proposed technique is at first illustrated on a 3-phase drive. Geometrical representations allow then to establish links with usual 3-phase SVM. The implementation of the proposed SVM is achieved in the vector control of a 5-phase drive. Experimental results are presented and confirm the theoretical approach. 


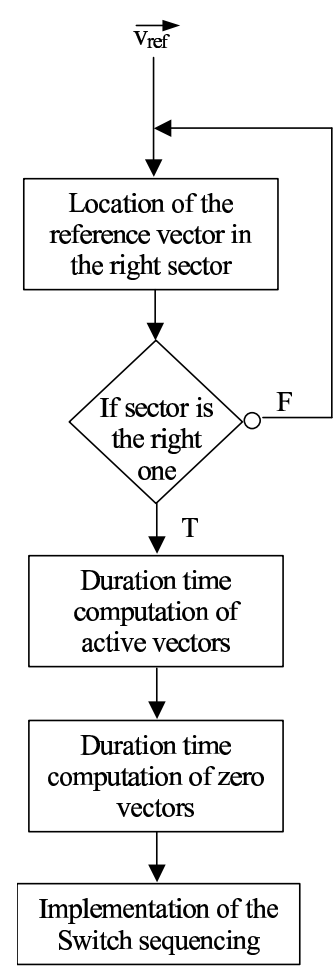

a)

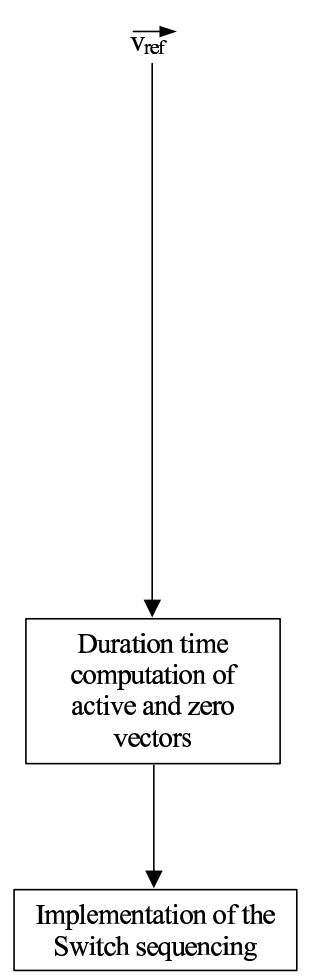

b)

Fig. 1. Classical a) and implemented b) SVM algorithms

\section{Multi-Phase SYSTEM SUPPliEd BY SVM VSI}

\section{A. Presentation of the two used topologies}

Multi-phase systems are usually wye connected to reduce the number of switches. Nevertheless, the $n$ phases of the system can be not electrically coupled to offer more reliability [21]. Figures 2 and 3 show the two topologies of supply.

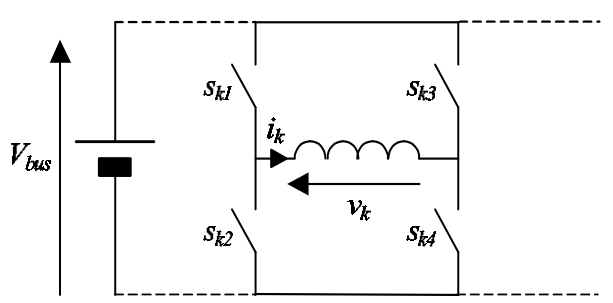

Fig. 2. No coupling topology: 2n legs required

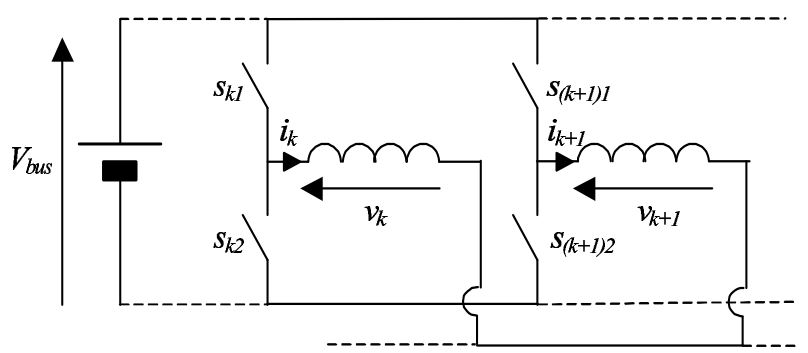

Fig. 3. Wye Connection topology: n legs required

\section{B. Vectorial modelling}

In an orthonormal base $B^{n}$ composed of the vectors $\left\{\overrightarrow{x_{1}^{n}}, \overrightarrow{x_{2}^{n}}, \ldots, \overrightarrow{x_{n}^{n}}\right\}$ the voltage vector can be defined as:

$$
\vec{v}=v_{1} \overrightarrow{x_{1}^{n}}+v_{2} \overrightarrow{x_{2}^{n}}+\ldots+v_{n} \overrightarrow{x_{n}^{n}}
$$

where $v_{k}$ is the voltage across the phase number $k$. 
If the VSI is a two-level one, $2^{n}$ different voltage vectors are generated.

The inductance matrix of a multi-phase machine is:

$$
\left[L_{s}^{n}\right]=\left(\begin{array}{cccc}
L_{11} & L_{12} & \ldots & L_{1 n} \\
L_{21} & L_{22} & \ldots & L_{2 n} \\
\vdots & \vdots & \ddots & \vdots \\
L_{n 1} & L_{n 2} & \ldots & L_{n n}
\end{array}\right)
$$

where $L_{k k}$ is the self inductance of the phase $k$ and $L_{j k}$ is the mutual inductance between phases $j$ and $k$.

The magnetic couplings between phases make difficult the control and it is more convenient to model the machine in a basis where there is no magnetic coupling.

In such a basis $B_{d}=\left\{\overrightarrow{x_{1}^{d}}, \overrightarrow{x_{2}^{d}}, \ldots, \overrightarrow{x_{n}^{d}}\right\}$, the new matrix inductance $\left[L_{s}^{d}\right]$ becomes:

$$
\left[L_{s}^{d}\right]=\left(\begin{array}{cccc}
\Lambda_{1} & 0 & \ldots & 0 \\
0 & \Lambda_{2} & \ldots & 0 \\
\vdots & \vdots & \ddots & \vdots \\
0 & 0 & \ldots & \Lambda_{n}
\end{array}\right)
$$

The linear relations between, $\Lambda$ and $L, \overrightarrow{x^{d}}$ and $\overrightarrow{x^{n}}$, are obtained by using an extended Concordia transformation.

In the basis $B_{d}$, we can define a set of fictitious magnetically independent systems associated to characteristic subspaces of $\left[L_{s}^{d}\right]$. This approach has been developed for a 5 -phase machine supplied by VSI [1]. The 5-phase drive can be considered as the association of 3 elementary fictitious machines supplied by 3 fictitious VSI (figure 4). The Multi-machine Multi-converter System concept [16] can be extended to systems with an arbitrary number of phases.

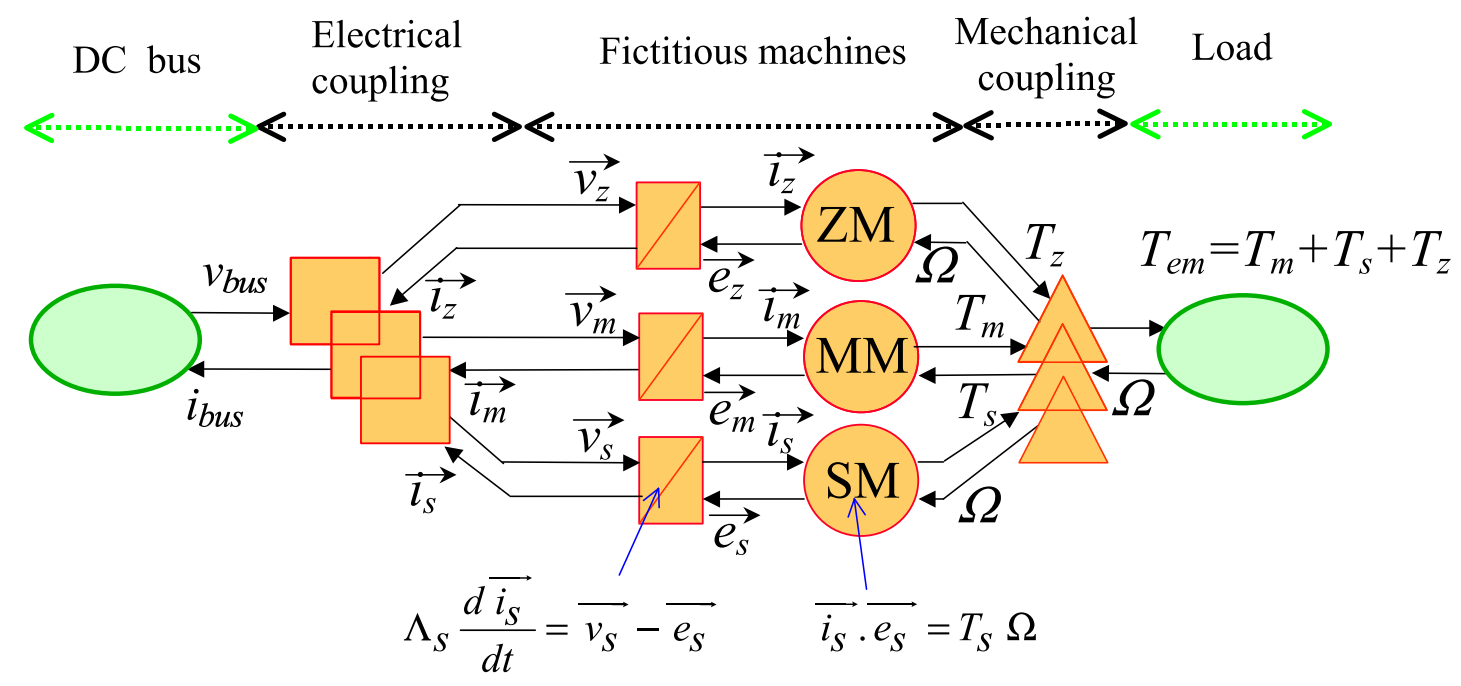

Fig. 4. Equivalence between a 5-phase machine and 3 fictitious machines supplied by 3 fictitious VSI.

In figure 4 , the three fictitious VSI generate three new voltage vectors $\overrightarrow{v_{z}}, \overrightarrow{v_{m}}$ and $\overrightarrow{v_{s}}$. These vectors are obtained by vectorial projections of $\vec{v}$ onto the three subspaces associated to the vectors of the base $B^{d}$. If instantaneous mode control of voltages is considered, there is a coupling between the three VSI. In an average mode control, the three VSI are independent. The last mode is considered in the paper. 
The average control laws give the three references corresponding to the vectors $\overrightarrow{v_{z}}, \overrightarrow{v_{m}}$ and $\overrightarrow{v_{s}}$. The duration times of active and zero vectors are obtained by vectorial computations. Then, switches duty cycles are deduced by very simple relations.

\section{Generation of an average voltage vector with consideration of switching constraints}

Many constraints can be imposed. For example, [2] and [15] have chosen instantaneous vectors to control dual three-phase induction machines and to reduce high harmonic currents, [4] and [6] to control 5-phase machines.

In this paper, we have chosen to control the number of switchings in a sampling period. Then, the following switching constraints are imposed:

- a minimum number of switchings are used to generate any initial average voltage reference;

- only one switching is possible at the same time.

A simple way to respect these two switching constraints is to make the same choice as a doublesided uniform-sampled pulse width modulation (UPWM):

- only $n+1$ different voltage vectors are used to generate any initial average voltage reference (a sufficient condition to generate any voltage reference);

- two voltage vectors consecutively generated by the VSI have only one different coordinate (there is then only one switching between two consecutive applied voltage vectors);

- each sequence of generated vectors begins and finishes with the same vector. A simple way is to repeat the sequence in the reverse order after it has been generated (there is then no switching between two periods of PWM).

The generated patterns are the same as UPWM but each conduction time of the vectors is computed with an extended SVM.

\section{Case of A three-Phase System}

\section{A. Introduction}

For example, let us choice a three-phase machine with no coupled phases. The choice of this system allows to establish the link between the proposed SVM and the classical one to control three-phase wye-connected machines. In an orthonormal basis $B^{n}$ composed of the vectors $\left\{\overrightarrow{x_{1}}, \overrightarrow{x_{2}}, \overrightarrow{x_{3}}\right\}$ the vectorial voltage equation of the machine is:

$$
\overrightarrow{v_{s}}=R_{s} \overrightarrow{i_{s}}+\left[\frac{d \overrightarrow{\phi_{s}}}{d t}\right]_{/ B^{n}}
$$

where $\overrightarrow{v_{s}}, \overrightarrow{i_{s}}$ and $\overrightarrow{\phi_{s}}$ are respectively the stator voltage, stator current and stator flux vectors.

Classically, the control of the machine is achieved in a Concordia frame. In other words, the control is carrying out in the basis $B^{d}=\left\{\overrightarrow{x_{z}}, \overrightarrow{x_{a}}, \overrightarrow{x_{b}}\right\}$, where there are no magnetic couplings, rather than in the natural basis $B^{n}$.

We remind the Concordia's transformation $C_{33}^{-1}$ :

$$
\left(\begin{array}{l}
\overrightarrow{x_{z}} \\
\overrightarrow{x_{a}} \\
\overrightarrow{x_{b}}
\end{array}\right)=\left(\begin{array}{ccc}
\frac{1}{\sqrt{3}} & \frac{1}{\sqrt{3}} & \frac{1}{\sqrt{3}} \\
\sqrt{\frac{2}{3}} & -\frac{1}{\sqrt{6}} & -\frac{1}{\sqrt{6}} \\
0 & +\frac{1}{\sqrt{2}} & -\frac{1}{\sqrt{2}}
\end{array}\right)\left(\begin{array}{l}
\overrightarrow{x_{1}} \\
\overrightarrow{x_{2}} \\
\overrightarrow{x_{3}}
\end{array}\right)
$$




\section{B. Modelling of the VSI}

Due to the two-level control $\left(v_{k}= \pm V_{b u s}\right)$, the voltage vector $\vec{v}=v_{1} \overrightarrow{x_{1}}+v_{2} \overrightarrow{x_{2}}+v_{3} \overrightarrow{x_{3}}$ can take $2^{3}=8$ different values. Fig. 5 shows the representation of the different values of $\vec{v}$ (blue crosses) in an orthonormal frame $\left\{O, \overrightarrow{x_{1}}, \overrightarrow{x_{2}}, \overrightarrow{x_{3}}\right\}$. Two values separated by only one switching are linked by a line. In this case, we obtain a cube.

To easily spot the different values of the vector $\vec{v}$, each value is associated with a number between 0 and 7 . This number is directly associated with the switching mode. When each decimal number is converted to a three-digit binary number, the 1's in this number indicates that the corresponding phase is supplied by $+V_{b u s}$ and the 0 's indicates that it is supplied by $-V_{\text {bus }}$. The most significant bit (first bit on the left) represents the switching state of phase 1 and so on.

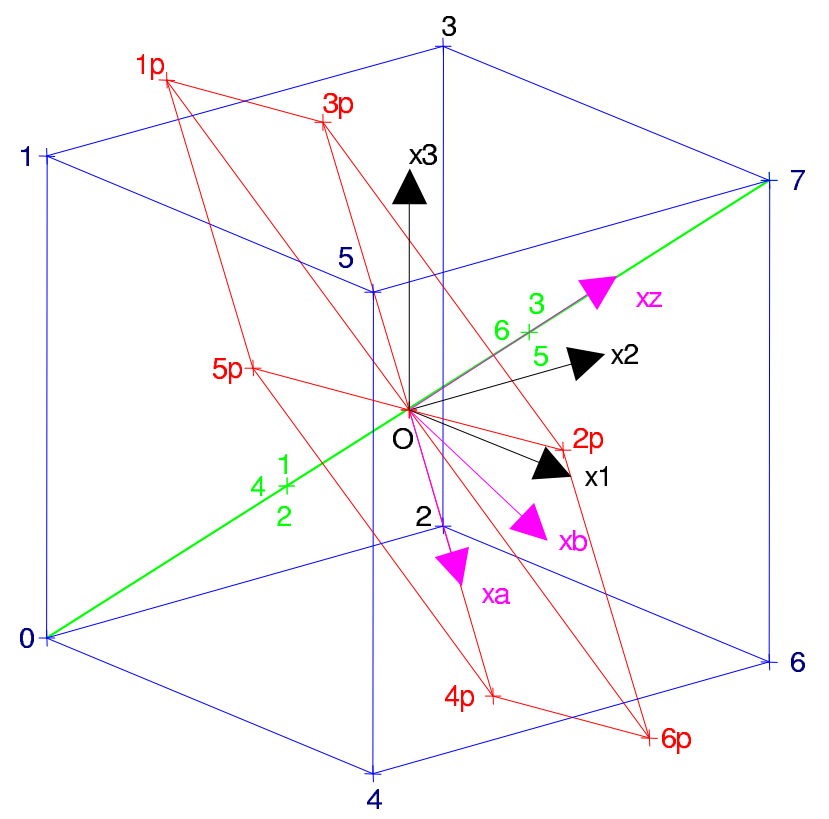

Fig. 5. 3D representation of the voltage vector and its projections onto particular subspaces

In the basis $B^{d}$, the stator inductance matrix has only two different values. The first one is associated with the vector $\overrightarrow{x_{z}}$, whose value is closed to leakage inductance value, and the second one is associated with the vectors $\overrightarrow{x_{a}}$ and $\overrightarrow{x_{b}}$, called synchronous inductance.

In the new basis $B^{d}$ it can be defined two particular orthogonal subspaces : a line supported by the vector $\overrightarrow{x_{z}}$ and a plane supported by the vectors $\overrightarrow{x_{a}}$ and $\overrightarrow{x_{b}}$.

Fig. 5 shows the line (in green), the plane (in red) and the projections of the $2^{3}$ values of the voltage vector onto.

It is important to notice that usually, machines are wye-connected and then only the plane has to be considered. In usual SVM, this plane is considered as a complex plane and the VSI is characterized by the hexagon $(1 \mathrm{p}, 2 \mathrm{p}, 3 \mathrm{p}, 4 \mathrm{p}, 5 \mathrm{p}, 6 \mathrm{p})$ that can be seen on Fig. 5.

\section{Generation of an average voltage}

The generation of the initial average voltage reference is now become generations of two references $\left\langle\overrightarrow{v_{z_{r e f}}}>\right.$ and $\left\langle\overrightarrow{v_{m_{\text {ref }}}}>\right.$, projections of $\left\langle\overrightarrow{v_{r e f}}>\right.$ respectively belonging to the line and the plane, such as:

$$
<\overrightarrow{v_{r e f}}>=<\overrightarrow{v_{z_{r e f}}}>+<\overrightarrow{v_{m_{r e f}}}>
$$


One solution to respect the switching constraints previously defined consists in using the family $(0,7)$ composed of groups of four vectors. In this family there are $3 !=6$ different combinations which respect the constraints (forming six identical tetrahedrons in the cube which are projected in six triangles onto the plane).

The classical technic needs to find the location of the vector into the 6 tetrahedrons (or the six triangles if there is a wye-coupling) and then to calculate the conduction time of the four vectors composing the chosen volume (or sector).

Due to the symmetry between the six tetrahedrons (or triangles), it is no more necessary to find this location.

If we take for example the combination of the vectors $\left(\begin{array}{llll}0 & 1 & 3 & 7\end{array}\right)$, the vector $\left\langle\overrightarrow{v_{r e f}}\right\rangle$ (wherever it is located into the cube) is given by the relation:

$$
<\overrightarrow{v_{r e f}}>=\alpha_{0}^{d} \overrightarrow{v_{0}}+\alpha_{1}^{d} \overrightarrow{v_{1}}+\alpha_{3}^{d \overrightarrow{v_{3}}}+\alpha_{7}^{d} \overrightarrow{v_{7}}
$$

Where the $\alpha_{k}^{d} \in[-1,1]$ can be positive or negative.

The aim is to write the vector $\left\langle\overrightarrow{v_{r e f}}>\right.$ in a way such as:

$$
<\overrightarrow{v_{\text {ref }}}>=V_{\text {bus }}\left(\left(2 \alpha_{1}^{n}-1\right) \overrightarrow{x_{1}}+\left(2 \alpha_{2}^{n}-1\right) \overrightarrow{x_{2}}+\left(2 \alpha_{3}^{n}-1\right) \overrightarrow{x_{3}}\right)
$$

where $\alpha_{k}^{n} \in[0,1]$ are the duty cycles of the VSI legs.

If we give the vectors $\overrightarrow{v_{0}}, \overrightarrow{v_{1}}, \overrightarrow{v_{3}}$ and $\overrightarrow{v_{7}}$ as an expression of $V_{b u s}, \overrightarrow{x_{1}}, \overrightarrow{x_{2}}$ and $\overrightarrow{x_{3}}$ we obtain:

$$
\begin{array}{ll}
\overrightarrow{v_{0}}=-V_{\text {bus }} \overrightarrow{x_{1}}-V_{\text {bus }} \overrightarrow{x_{2}}-V_{\text {bus }} \overrightarrow{x_{3}} & \overrightarrow{v_{1}}=-V_{\text {bus }} \overrightarrow{x_{1}}-V_{\text {bus }} \overrightarrow{x_{2}}+V_{\text {bus }} \overrightarrow{x_{3}} \\
\overrightarrow{v_{3}}=-V_{\text {bus }} \overrightarrow{x_{1}}+V_{\text {bus }} \overrightarrow{x_{2}}+V_{\text {bus }} \overrightarrow{x_{3}} & \overrightarrow{v_{7}}=+V_{\text {bus }} \overrightarrow{x_{1}}+V_{\text {bus }} \overrightarrow{x_{2}}+V_{\text {bus }} \overrightarrow{x_{3}}
\end{array}
$$

These four equations placed in equation (1) give:

$$
<\overrightarrow{v_{r e f}}>=V_{\text {bus }}\left(\left(-\alpha_{0}^{d}-\alpha_{1}^{d}-\alpha_{3}^{d}+\alpha_{7}^{d}\right) \overrightarrow{x_{1}}+\left(-\alpha_{0}^{d}-\alpha_{1}^{d}+\alpha_{3}^{d}+\alpha_{7}^{d}\right) \overrightarrow{x_{2}}+\left(-\alpha_{0}^{d}+\alpha_{1}^{d}+\alpha_{3}^{d}+\alpha_{7}^{d}\right) \overrightarrow{x_{3}}\right)
$$

As the four vectors $\overrightarrow{v_{0}}, \overrightarrow{v_{1}}, \overrightarrow{v_{3}}$ and $\overrightarrow{v_{7}}$ are used in a period of PWM, we have the relation:

$$
\alpha_{0}^{d}+\alpha_{1}^{d}+\alpha_{3}^{d}+\alpha_{7}^{d}=1
$$

Relation (4) in equation (3) gives:

$$
<\overrightarrow{v_{r e f}}>=V_{\text {bus }}\left(\left(2 \alpha_{7}^{d}-1\right) \overrightarrow{x_{1}}+\left(2\left(\alpha_{3}^{d}+\alpha_{7}^{d}\right)-1\right) \overrightarrow{x_{2}}+\left(2\left(\alpha_{1}^{d}+\alpha_{3}^{d}+\alpha_{7}^{d}\right)-1\right) \overrightarrow{x_{3}}\right)
$$

By identification of the equations (2) and (5) we can finally find:

$$
\begin{aligned}
& \alpha_{1}^{n}=\alpha_{7}^{d} \\
& \alpha_{2}^{n}=\alpha_{3}^{d}+\alpha_{7}^{d} \\
& \alpha_{3}^{n}=\alpha_{1}^{d}+\alpha_{3}^{d}+\alpha_{7}^{d}
\end{aligned}
$$

\section{Computation of the $\alpha_{k}^{d}$ 's}

To calculate an $\alpha_{k}^{d}$ it is sufficient to achieve mixed products:

$$
\begin{array}{ll}
\alpha_{7}^{d}=\frac{\left(\overrightarrow{v_{0}}\left|\overrightarrow{v_{1}}\right| \overrightarrow{v_{3}} \mid<\overrightarrow{v_{r e f}}>\right)}{\left(\overrightarrow{v_{0}}\left|\overrightarrow{v_{1}}\right| \overrightarrow{v_{3}} \mid \overrightarrow{v_{7}}\right)} \quad \alpha_{3}^{d}=\frac{\left(\overrightarrow{v_{0}}\left|\overrightarrow{v_{1}}\right|<\overrightarrow{v_{r e f}}>\mid \overrightarrow{v_{7}}\right)}{\left(\overrightarrow{v_{0}}\left|\overrightarrow{v_{1}}\right| \overrightarrow{v_{3}} \mid \overrightarrow{v_{7}}\right)} \\
\alpha_{1}^{d}=\frac{\left(\overrightarrow{v_{0}}\left|<\overrightarrow{v_{r e f}}>\right| \overrightarrow{v_{3}}\right)}{\left(\overrightarrow{v_{0}}\left|\overrightarrow{v_{1}}\right| \overrightarrow{v_{3}} \mid \overrightarrow{v_{7}}\right)} \quad \alpha_{0}^{d}=\frac{\left(<\overrightarrow{v_{r e f}}>\left|\overrightarrow{v_{1}}\right| \overrightarrow{v_{3}} \mid \overrightarrow{v_{7}}\right)}{\left(\overrightarrow{v_{0}}\left|\overrightarrow{v_{1}}\right| \overrightarrow{v_{3}} \mid \overrightarrow{v_{7}}\right)}
\end{array}
$$

The calculation of a mixed product is the same as a determinant and requires only a few sums and products. The denominator is constant and can be calculated off-line. The numerator has only one variable, $\left\langle\overrightarrow{v_{r e f}}\right\rangle$, and can be reduced to few calculations. 


\section{E. Experimental results}

The experimental test bench is composed of a VSI controlled by a dSPACE DS1103 PPC Controller Board. The control board operates in a host PC uses as PWM controller the TMS320F240 16-bit fixed-point DSP and is programmed under MATLAB-SIMULINK.

Figures 6 show the currents in the real and the fictitious machines obtained with the use of an open-loop U/f control.
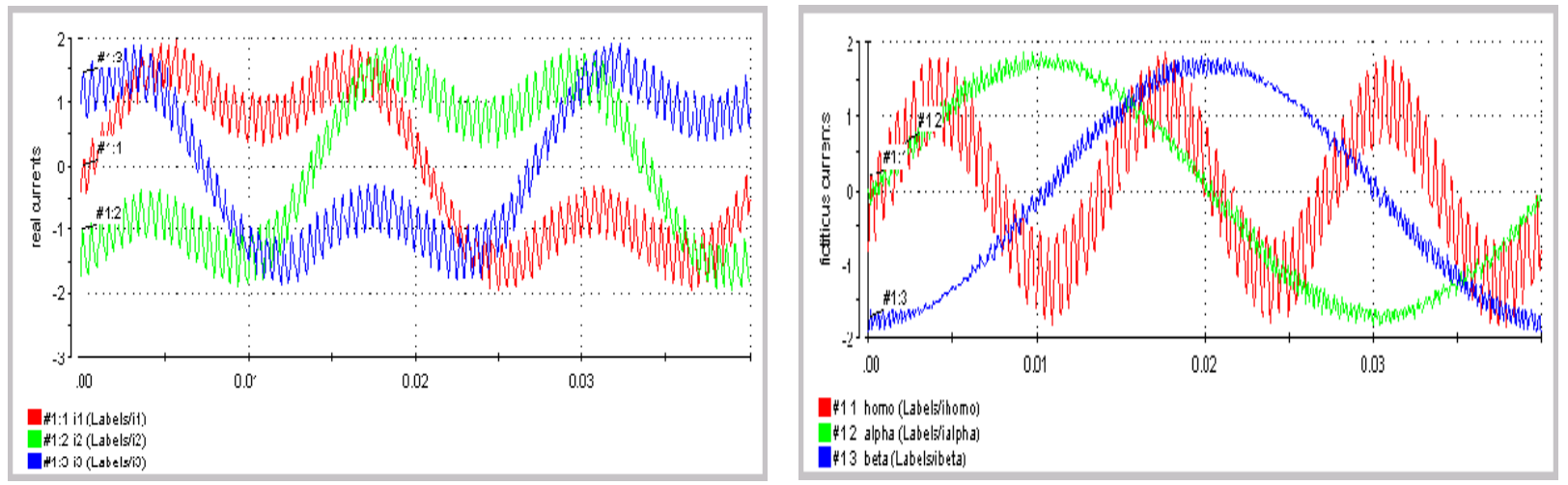

Fig. 6. Practical results.

Only five multiplications and seven additions are necessary to generate currents in the main and the zero-sequence machine with respect of switching constraints.

\section{CASE OF A FIVE-PHASE SYSTEM}

\section{A. Introduction}

To reduce the number of switches, we choose to wye-connect the phases of the machine. The basis $B^{d}$ where there are no magnetic couplings is deduced from the extended Concordia's transformation below:

$$
\left(\begin{array}{c}
\overrightarrow{x_{z}} \\
\overrightarrow{x_{a}} \\
\overrightarrow{x_{b}} \\
\overrightarrow{x_{c}} \\
\overrightarrow{x_{d}}
\end{array}\right)=\left(\begin{array}{ccccc}
\frac{1}{\sqrt{5}} & \frac{1}{\sqrt{5}} & \frac{1}{\sqrt{5}} & \frac{1}{\sqrt{5}} & \frac{1}{\sqrt{5}} \\
\sqrt{\frac{2}{5}} & \sqrt{\frac{2}{5}} \cos \frac{2 \pi}{5} & \sqrt{\frac{2}{5}} \cos \frac{4 \pi}{5} & \sqrt{\frac{2}{5}} \cos \frac{6 \pi}{5} & \sqrt{\frac{2}{5}} \cos \frac{8 \pi}{5} \\
0 & \sqrt{\frac{2}{5}} \sin \frac{2 \pi}{5} & \sqrt{\frac{2}{5}} \sin \frac{4 \pi}{5} & \sqrt{\frac{2}{5}} \sin \frac{6 \pi}{5} & \sqrt{\frac{2}{5}} \sin \frac{8 \pi}{5} \\
\sqrt{\frac{2}{5}} & \sqrt{\frac{2}{5}} \cos \frac{4 \pi}{5} & \sqrt{\frac{2}{5}} \cos \frac{8 \pi}{5} & \sqrt{\frac{2}{5}} \cos \frac{12 \pi}{5} & \sqrt{\frac{2}{5}} \cos \frac{16 \pi}{5} \\
0 & \sqrt{\frac{2}{5}} \sin \frac{4 \pi}{5} & \sqrt{\frac{2}{5}} \sin \frac{8 \pi}{5} & \sqrt{\frac{2}{5}} \sin \frac{12 \pi}{5} & \sqrt{\frac{2}{5}} \sin \frac{16 \pi}{5}
\end{array}\right)\left(\begin{array}{l}
\overrightarrow{x_{1}} \\
\overrightarrow{x_{2}} \\
\overrightarrow{x_{3}} \\
\overrightarrow{x_{4}} \\
\overrightarrow{x_{5}}
\end{array}\right)
$$

Due to the five dimensions, it is no more possible to represent graphically the different values of the voltage vectors in the natural basis. Three orthogonal subspaces can be defined:

- a line associated with the vector $\overrightarrow{x_{z}}$;

- a plane called main associated with the vectors $\overrightarrow{x_{a}}$ and $\overrightarrow{x_{b}}$;

- a plane called secondary associated with the vectors $\overrightarrow{x_{c}}$ and $\overrightarrow{x_{d}}$.

The $2^{5}$ projections of the voltage vectors onto these subspaces are shown in figures 7 and 8 . The line is not considered due to the wye connection.

The voltage vector reference is then:

$$
<\overrightarrow{v_{r e f}}>=<\overrightarrow{v_{z_{\text {ref }}}}>+<\overrightarrow{v_{m_{\text {ref }}}}>+<\overrightarrow{v_{s_{\text {ref }}}}>
$$

where $\left\langle\overrightarrow{v_{z_{\text {ref }}}}>=\overrightarrow{0},\left\langle\overrightarrow{v_{m_{\text {ref }}}}>\right.\right.$ belongs to the main plane and $\left\langle\overrightarrow{v_{s_{r e f}}}>\right.$ to the secondary plane. 


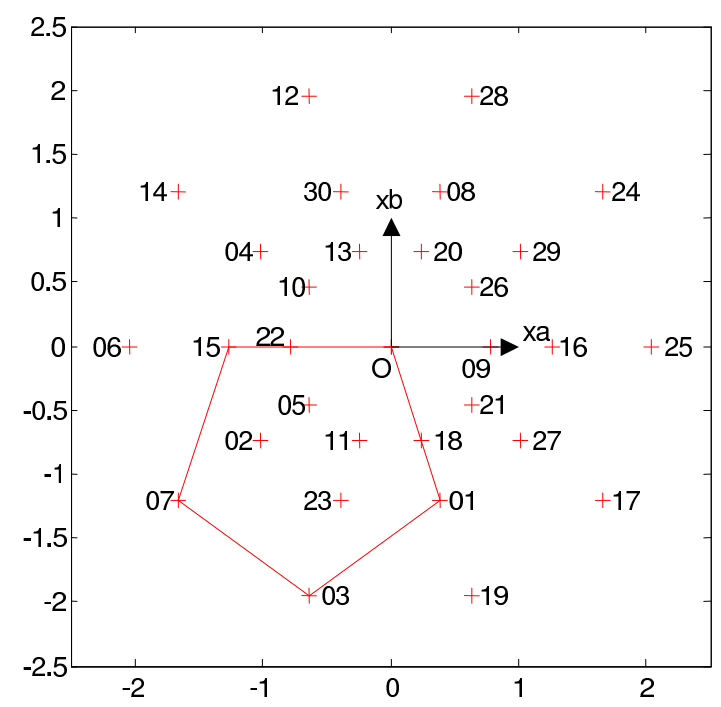

Fig. 7. Projections of the voltage vector on the main plane

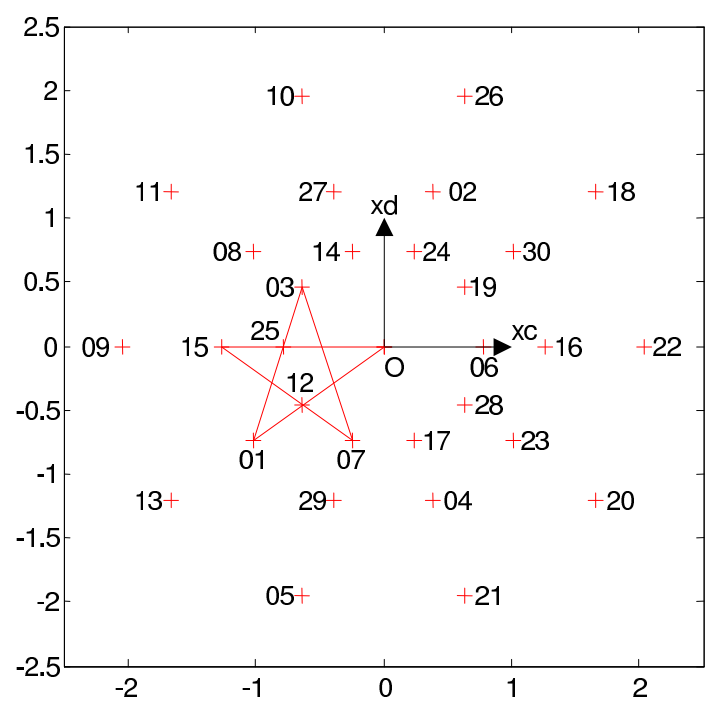

Fig. 8. Projections of the voltage vector on the secondary plane

\section{B. Computation of the duty cycles}

As for the three-phase system, a choice consists in using the family $(0,31)$. In this family, there are $5 !=120$ different combinations of six vectors which respect the switching constraints.

If we take for example the combination of the vectors ( $\left.\begin{array}{l}0 \\ 0\end{array} 371531\right)$ shown in red in figures 7 and 8 , the vector $\left\langle\overrightarrow{v_{r e f}}>\right.$ to be synthetized is given by the relation:

$$
<\overrightarrow{v_{r e f}}>=\alpha_{0}^{d} \overrightarrow{v_{0}}+\alpha_{1}^{d} \overrightarrow{v_{1}}+\alpha_{3}^{d} \overrightarrow{v_{3}}+\alpha_{7}^{d} \overrightarrow{v_{7}}+\alpha_{15}^{d} \overrightarrow{v_{15}}+\alpha_{31}^{d} \overrightarrow{v_{31}}
$$

By the same way as previously, we can find the relations:

$$
\begin{aligned}
& \alpha_{1}^{n}=\alpha_{31}^{d} \\
& \alpha_{2}^{n}=\alpha_{15}^{d}+\alpha_{31}^{d} \\
& \alpha_{3}^{n}=\alpha_{7}^{d}+\alpha_{15}^{d}+\alpha_{31}^{d} \\
& \alpha_{4}^{n}=\alpha_{3}^{d}+\alpha_{7}^{d}+\alpha_{15}^{d}+\alpha_{31}^{d} \\
& \alpha_{5}^{n}=\alpha_{1}^{d}+\alpha_{3}^{d}+\alpha_{7}^{d}+\alpha_{15}^{d}+\alpha_{31}^{d}
\end{aligned}
$$

\section{Experimental results}

This algorithm of computation has been used in a vector current control of a 5-phase DCbrushless machine. The switches are controlled with a the same DSPACE board as previously. Experimental bench is shown in figure 9, control structure of the system in figure 10. Figures 11 and 12 show the currents in the real and the fictitious machines with the following references:

$$
\begin{aligned}
& \overrightarrow{i_{m_{r e f}}}=I_{m}\left(\sin (2 \pi 10 t) \overrightarrow{x_{a}}+\sin \left(2 \pi 10 t+\frac{\pi}{2}\right) \overrightarrow{x_{b}}\right) \\
& \overrightarrow{i_{s_{r e f}}}=I_{s}\left(\sin (2 \pi 30 t) \overrightarrow{x_{c}}+\sin \left(2 \pi 30 t+\frac{3 \pi}{2}\right) \overrightarrow{x_{d}}\right)
\end{aligned}
$$

With a little more computations compared with the three-phase case (six multiplications and fifteen additions), fast algorithm allows to generate currents in the main and the principal machines with respect of switching constraints. 


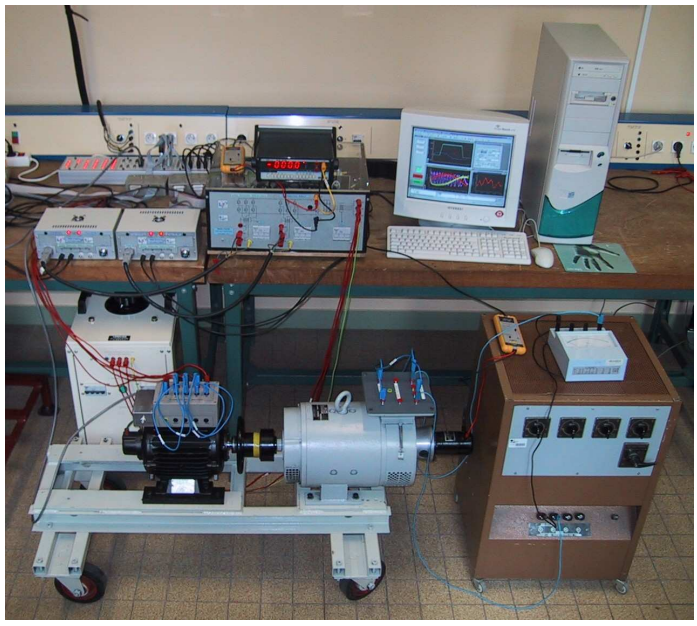

Fig. 9. Experimental bench

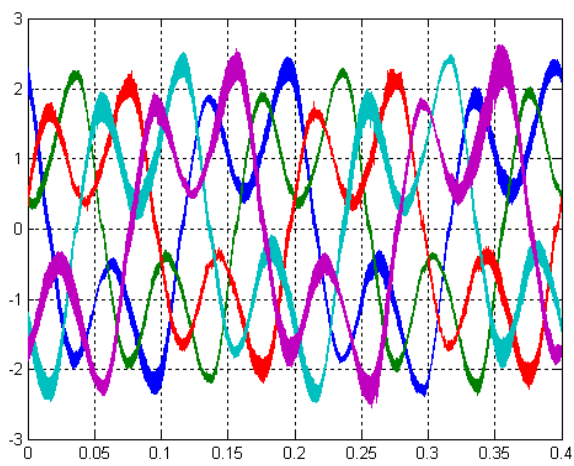

Fig. 11. Currents of the 5 phases

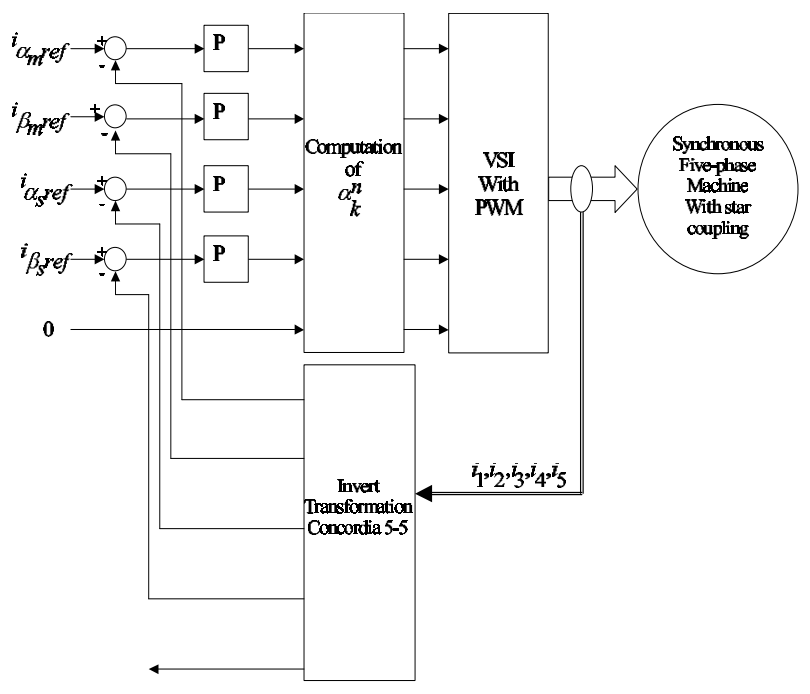

Fig. 10. Structure of the proposed control

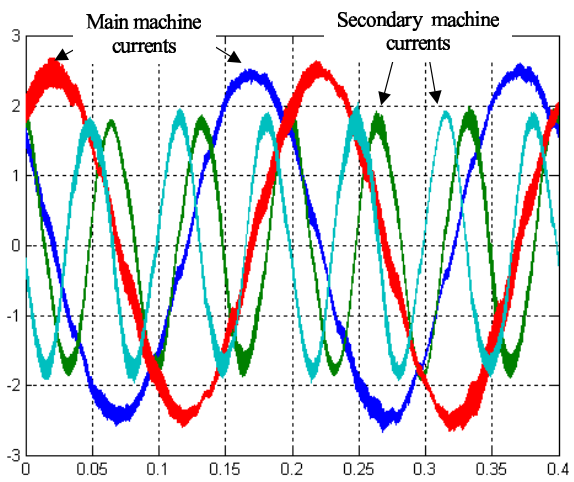

Fig. 12. Currents of fictitious machines

\section{Conclusion}

This paper show a new fast SVM algorithm to compute the duty cycles of VSI's legs. This SVM algorithm does not need to locate the vector in a particular sector and can be implemented with a low capability computation system. Consequently, with the proposed approach, the drawback of prohibitive time computation of SVM for multi-phase systems disappears. We found out the interest of SVM as explicit computation of every duty cycles of the VSI legs and possibility to take into account saturation effects.

Particular patterns of PWM are used to take into account switching constraints. Other constraints could be chosen for example to generate less harmonic currents in the secondary machines.

This work is based on a vectorial modelling of multi-phase systems associated with the Multimachine Multi-converter System approach which permits the extension to an arbitrary number of phases and to three-level inverters. 


\section{REFERENCES}

[1] X. Kestelyn, E. Semail, J.P Hautier, Vectorial Multi-machine Modeling for a 5-Phase Machine, ICEM Brugges, August 2002, CD-ROM.

[2] Z.Yifan, T. A. Lipo, Space Vector PWM Control of Dual Three-Phase Induction Maching Using Vector Space Decomposition, IEEE Transactions on Industry Applications, Vol. 31. no 5, September/October 1995, pp. 1100-1109.

[3] E. Semail, Tools and studying method of polyphase electrical systems, Generalization of the space vector theory, Ph.D. thesis USTL(University of Sciences and Technologies of Lille), June 2000 (text in French).

[4] H.A. Toliyat, S. Ruhe, X. Huangsheng, A DSP-Based Vector Control of 5-Phase Synchronous Reluctance Motor, IEEE-IAS annual meeting 2000, Rome, october 2000, CD-ROM.

[5] E. Semail, C. Rombaut New tools for studying voltage-source inverters, IEEE Power Engineering Review, Vol. 22. no 3, March 2002, pp. 47-48

[6] J. P. Martin , E. Semail , S. Pierfederici,A. Bouscayrol, F. Meibody-Tabar , B. Davat, Space Vector Control of 5-phase PMSM supplied by q H-bridge VSIs, ElectrIMACS 2002, Montreal, August 2002, CDROM.

[7] E. Semail , C. Rombaut, New method to calculate the conduction durations of the switches in a n-leg 2-level Voltage Source, EPE 2001, 27-29 August, 2001, Graz, Austria,CDROM.

[8] D. Gondouin, F. Menneron New diesel-electric propulsion system topologies, AES2000, October 2000, Paris, pp. 66-71.

[9] A. Hava, R. Kerkman ,T. Lipo, Carrier-basisd PWM VSI overmodulation strategies: analysis, comparison and design, IEEE Trans. on Power Electronics, vol. 13, no. 4, July 1998, pp. 624-689.

[10] W. Leonhard., 30 years space vectors, 20 years field orientation, 10 years digital signal processing with controlled $A C$ drives, EPE Journal, vol. 1, no. 1, July 1991, pp. 13-20.

[11] J. Holtz, Pulse width modulation - A survey, IEEE Trans. on Industrial Electronics, vol. 39 no. 5 , December 1992, pp. 410-419.

[12] V. Blasko Analysis of a hybrid PWM basisd on modified space vector and triangle-comparison methods, IEEE Transactions on industry applications, Vol. 33, No. 1, May/June 1997,pp. 756-764.

[13] E. Semail, A. Bouscayrol,J.P. Hautier, Vectorial formalism for analysis and design of polyphase synchronous machines, EPJ Applied Physics, EPJ AP (European Physical Journal-Applied Physics), Vol. 22, no. 3, June 2003, pp. 207-220.

[14] P. Delarue,A. Bouscayrol ,E. Semail Generic Control Method of Multileg Voltage-Source-Converters for Fast Practical Implementation, IEEE Trans. on Power Electronics, vol. 18, no. 2, March 2003, pp. 517-526.

[15] D. Hadiouche,H. Razik,A. Rezzoug Modelling of a Double Star Induction Motor for Space Vector PWM Control, ICEM 2000, August 2000, Espoo (Finland), pp. 392-396.

[16] A. Bouscayrol, B. Davat, B. de Fornel, B. François, J. P. Hautier, F. Meibody-Tabar, M. PietrzakDavid. Multi-machine Multi-converter System: application for the electromechanical drives, EPJ Applied Physics, Vol. 10, no. 2, May 2000, pp-131-147, (common paper of GREEN, L2EP and LEEI, according to the MMS project of the French GdR-SDSE).

[17] Bhatia R., Krattiger H., Bonanini A., Schafer D., Inge J.T., Sydnor G.H. Adjustable Speed Drive With A Single 100-MW Synchronous Motor ABB Review Issue, No6, 1998, pp.14-20.

[18] H. Godfroi, P. Bosc. Large variable speed drives using synchronous motors and frequency converters Alstom Review nř6, 1986.

[19] S. Siala S., E. Guette, J. L. Pouliquen. Multi-inverter PWM control: a new generation drives for cruise ship electric propulsion, European Power Electronics Conference (EPE2003), Toulouse (France), CDROM.

[20] D. Beliaev, A. Weinger, B. Ingram, F. DeWinter. Polyphase Systems For High-Power Controlled AC, European Power Electronics Conference (EPE2003), Toulouse (France), CD-ROM.

[21] T.M Jahns. Improved reliability in solid-state ac drives by means of multiple independent phase-drive units, IEEE Transactions on Industry Applications, Vol. IA16, pp 321-331, May/June 1980. 


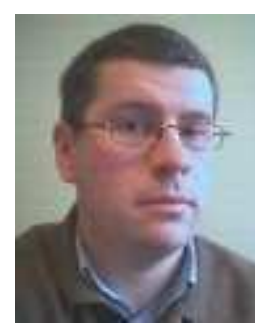

Xavier Kestelyn was born in France in 1971. He received the teaching degree "Agrégation" in 1997 and Ph.D. degree in electrical engineering in 2003 in University of Sciences and Technology of Lille (France). He is a member of the Multi-Machine Multi-Converter System national French project of research. His fields of interest include Modelling, Control and Design of Polyphase Systems (Converters and AC Drives).

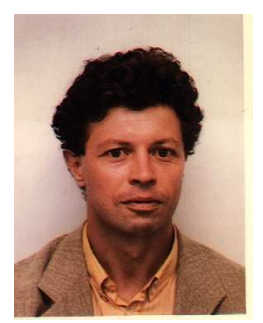

Eric Semail is graduated from the Ecole Normale Supérieure, Cachan, France. He received the teaching degree "Agrégation" in 1986. From 1987 to 2001, he has been professor (holder of agrégation) in University of Lille (USTL). He received Ph.D. degree in 2000 and became associate professor at ENSAM Lille in 2001. In L2EP (Laboratory of Electrical Engineering of Lille) his fields of interest include modeling, control and design of polyphase systems (converters and AC Drives)

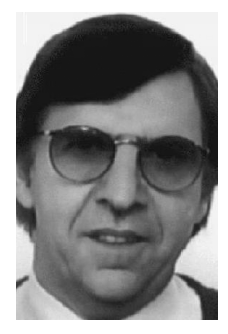

Jean-Paul Hautier received the Ph.D. degree from the University of Lille (USTL), France, in 1984. He received the "Habilitation à diriger des Recherches" degree from Engineering School of Douai, France, in 1989. In 1989, he became Professor at the ENSAM Lille. Its main research interests are power electronics and electrical systems control. Since 2000, Pr. Hautier has the head of the the L2EP Lille (Laboratory of Electrical Engineering of Lille). 